\title{
The impact of chemotherapy on persistent ground-glass nodules in patients with lung adenocarcinoma
}

\author{
Wenwen Lu ${ }^{1,2}$, Matthew D. Cham ${ }^{3}$, Linlin $\mathrm{Qi}^{1}$, Jianwei Wang ${ }^{1}$, Wei Tang ${ }^{1}$, Xiaolu Li ${ }^{1}$, Jie Zhang ${ }^{4}$ \\ ${ }^{1}$ Department of Diagnostic Radiology, National cancer center, Cancer Hospital/Institute, Chinese Academy of Medical Sciences/Peking Union \\ Medical College, Beijing 100021, China; ${ }^{2}$ Peking University Eye Center, The Third Hospital of Peking University, Beijing 100191, China; \\ ${ }^{3}$ Department of Radiology Box 1234/Icahn School of Medicine at Mount Sinai, New York, USA; ${ }^{4}$ Radiology Department, Dongzhimen Hospital/ \\ Beijing University of Chinese Medicine, Beijing 100700, China \\ Contributions: (I) Conception and design: J Wang; (II) Administrative support: None; (III) Provision of study materials or patients: J Wang; (IV) \\ Collection and assembly of data: W Lu, L Qi; (V) Data analysis and interpretation: W Tang, X Li, J Zhang; (VI) Manuscript writing: All authors; (VII) \\ Final approval of manuscript: All authors. \\ Correspondence to: Jianwei Wang, MD. Department of Diagnostic Radiology, National cancer center, Cancer Hospital/Institute, Chinese Academy of \\ Medical Sciences/Peking Union Medical College, Beijing 100021, China. Email: dr_jianweiwang@sina.com.
}

Backgrounds: To evaluate the response of persistent ground glass nodules (GGNs) in patients with lung adenocarcinoma treated with platinum-based chemotherapy on computed tomography (CT).

Methods: We retrospectively studied patients with GGNs that met the following criteria: (I) GGNs found in patients with lung adenocarcinoma, which persist for more than 3 months; (II) patients treated with platinum-based (cisplatin or carboplatin) chemotherapy for at least 2 cycles; (III) ground glass proportion $\geq 50 \%$. For each patient, if more than two CTs satisfied the inclusion criteria, then the baseline and last CTs were used for analysis, defined as CT1 and CT2. A total of 91 persistent pulmonary GGNs in 51 patients fulfilled the inclusion criteria. We defined growth as a nodule $\geq 2 \mathrm{~mm}$ increase in diameter or showing up a solid portion. GGN response to therapy was assessed and compared with the baseline CT. Differences in CT findings were analyzed using a paired $t$-test and Pearson $\chi^{2}$ test.

Results: Between 2010 and 2015, 25 of the 51 (49\%) were male and 26 of the 51 (51\%) were female. The average age at time of detection of a GGN was 63.8 (range, 36-84) years. Mean follow-up duration was $24.1 \pm 17.9$ months. During the follow-up periods, on a per-nodule basis, $94.5 \%$ of GGNs ( $=86$ ) remained unchanged in size. Only $5.5 \%$ GGNs $(n=5)$ in 5 patients increased in size. The nodules CT feature in each lung adenocarcinoma clinical stage show no difference. No significant difference was found in the size, attenuation, volume, and mass of GGN between baseline and post-treatment measurements, regardless of the type of chemotherapy $(\mathrm{P}>0.05)$.

Conclusions: The clinical course of GGNs in patients with lung adenocarcinoma is predominantly indolent, and platinum-based chemotherapy may have no effect on the growth of persistent GGNs.

Keywords: Ground-glass nodules (GGNs); lung cancer; adenocarcinoma; chemotherapy

Submitted Aug 05, 2017. Accepted for publication Oct 07, 2017.

doi: $10.21037 /$ jtd.2017.10.50

View this article at: http://dx.doi.org/10.21037/jtd.2017.10.50 


\section{Introduction}

Cases of lung cancer involving ground-glass nodules (GGNs) have been detected with increasing frequency since the advent of computed tomography (CT). A GGN is a circumscribed lung nodule characterized by ground glass opacification (GGO), which in turn is defined as hazy increased attenuation of lung with preservation of vascular and bronchial margins on CT $(1,2)$. In the literature, GGN and GGO have often been used interchangeably although the former is more widely used in recent years $(3,4)$. GGNs are strongly associated with lung neoplasms that have a lepidic growth pattern and there is a well-established relationship between GGNs and the spectrum of neoplasms formerly known as bronchioloalveolar carcinoma (BAC) (5-9). Investigations into the natural history of GGNs have revealed two major subgroups: ones that need treatment and others that may be observed for a long time (10).

A past history of lung cancer has also been reported to influence GGN growth. Hiramatsu et al. (11) analyzed 125 GGNs in 125 patients using logistic regression model, finding that a past history of lung cancer was a statistically significant predictor of GGN growth. Close monitoring of the concurrent GGNs during CT follow-up is necessary to evaluate for malignant features, such as an increase in overall size or the development of a solid component within the GGNs, in addition to looking for primary lung cancer recurrence $(12,13)$. Little is known about the proliferation rate or molecular mechanisms of GGNs $(6,12,14,15)$. Despite satisfactory outcomes after complete resection of adenocarcinoma in situ (AIS) (16-18), recurrence at the cut-end has been reported even after complete resection with negative margins $(13,19,20)$. Guidelines on the management of incidentally detected GGNs suggest that a minimum follow-up duration of 3 to 4 years is required to establish an indolent growth pattern (3). However, there are no guidelines pertaining to the maximum follow-up duration that would definitively rule out future malignant transformation, or when follow-up may be safely stopped. This level of uncertainty, coupled with perpetual patient anxiety, raises the importance of managing these lesions in a more definitive manner. The decision to observe carefully or treat small GGNs is not well established, especially in patients with a history of lung cancer or synchronous multiple primary lung cancer. Surgical treatment may not be practical in patients with a reduced pulmonary reserve after having undergone lung resection for their primary tumors. Many clinicians may consider chemotherapy as an alternative treatment for those lung cancers manifesting as multiple GGNs. Is it actually effective? In this study, we retrospectively reviewed the records of a surgical series of lung cancer patients with solitary or synchronous multiple lung GGNs, in an attempt to identify whether chemotherapy has an impact on their size and growth. To the best of our knowledge, this is the first report in the literature describing the effect of chemotherapy on persistent GGNs.

\section{Methods}

The institutional review boards of our hospitals approved this retrospective study and informed consent was waived. Between 2010 and 2015, we identified 91 persistent pulmonary GGNs in 51 patients with lung adenocarcinoma who underwent platinum-based chemotherapy (cisplatin or carboplatin) for at least 2 cycles. Persistent GGNs, defined as GGNs that exist for at least 3 months, were identified during CT of patients with lung adenocarcinoma. The follow-up duration was defined as the period from the date of GGN identification by CT (CT1) to the date of the most recent CT (CT2). Patients whose follow-up periods were shorter than 6 months, had confirmed pulmonary metastasis, progression of other malignancies, or lost to follow-up were excluded.

\section{Radiological definition}

All CT scans (include plain CT and enhanced CT) were performed at a single institution using 1.0 to $1.25 \mathrm{~mm}$ thick sections with or without contrast enhancement. The parameters of CT scan kept the same among the baseline CT and follow-up CT in each patient. CT scans were reviewed at a window level of -600 Hounsfield units (HU) and window width of $1,500 \mathrm{HU}$ on computer workstations. During CT examination process, the two inspiratory states differ greatly are need to be excluded. Two experienced radiologists reviewed each CT scan independently for the purposes of this study. Both radiologists were blinded to the patients' detailed clinical information but they knew that the patients had a history of lung cancer. In cases of discordant interpretations, decisions on CT findings were reached by consensus. As a measure of size, the diameter of a nodule was defined as the average of its maximal length and maximal orthogonal diameter, which were measured with electronic calipers on the image that showed the largest cross-sectional area of the nodule. The height of the nodule 
Table 1 Formulas

\begin{tabular}{lll}
\hline Variable name & Formulas & Detail \\
\hline Volume $\left(\mathrm{mm}^{3}\right)$ & $\Pi / 6 \times \mathrm{D} \times \mathrm{d} \times \mathrm{h}$ & $\begin{array}{l}\text { D: maximal axial diameter of nodule; } \mathrm{d} \text { : maximal orthogonal diameter (axial slice); } \\
\text { h: nodule maximal height }\end{array}$ \\
Mass (g) (13) & Volume $\times(1,000+\mathrm{DHU}) / 10^{6}$ & DHU: average density in HU volume in $\mathrm{mm}^{3}$ \\
\hline
\end{tabular}

HU, Hounsfield unit.

Table 2 Characteristics of 91 GGNs

\begin{tabular}{lcc}
\hline Factor & Category & Number of GGNs (\%) \\
\hline Lesion average size & $\leq 1 \mathrm{~cm}$ & $60(65.9)$ \\
& $>1 \mathrm{~cm}$ & $31(34.1)$ \\
Inner solid component & pGGN & $87(95.6)$ \\
& mGGN & $4(4.4)$ \\
Lesion number & Single & $32(62.7)$ \\
& Multiple & $19(37.3)$ \\
\hline
\end{tabular}

GGNs, ground glass nodules; pGGN, pure ground-glass nodule; mGGN, mixed ground-glass nodule.

was also measured on a coronal reconstruction. Nodule attenuation was evaluated by placing the largest possible circular region of interest (ROI) in the nodule using the transverse slice that showed the largest cross-sectional area on soft kernel reconstructions and excluding any visible vessel(s) within the nodule, each nodule is measured three times and then take the averages. Nodule volume and mass were calculated using diameters from manual measurements (21). Corresponding formulas are summarized in Table 1. Other CT findings of each nodule were analyzed as follows: shape (regular or irregular), vacuole, pleural indentation. We defined growth as a nodule $\geq 2 \mathrm{~mm}$ increase in diameter, based on a prior report by Kakinuma et al. stating that a diameter increase of $>1.72 \mathrm{~mm}$ is necessary to reliably identify growth considering interobserver measurement error (22).

The initial CT scans were performed prior to the initial cycle of chemotherapy, and the follow-up CT scans were conducted after at least two cycles of platinum-based chemotherapy. Statistical analyses were performed using the SPSS 19.0.

The effect of therapy on nodule CT findings (average size, CT attenuation value, volume and mass) was evaluated by using a paired $t$-test, GGN CT feature (shape, vacuole and pleural retraction) were compared among the each clinical stage of lung adenocarcinoma by using the Pearson $\chi^{2}$ test. Significance was accepted at values of $\mathrm{P}$ less than 0.05 .

\section{Results}

Fifty-one patients with 91 GGNs met the inclusion criteria, 25 were male $(49 \%)$ and 26 were female $(51 \%)$. The average age at time of detection of a GGN was 63.8 years (range, 36-84 years). All patients underwent either cisplatin or carboplatin chemotherapy.

Out of the 91 GGNs, 60 nodules $(65.9 \%)$ were less than or equal to $1 \mathrm{~cm}, 87$ (95.6\%) were pure ground glass, and 19 $(37.3 \%)$ were multiple (Table 2). Mean follow-up duration was $24.1 \pm 17.9$ months.

During the follow-up period, on a per-nodule basis, 86 (94.5\%) of GGNs remained unchanged in size (Figure 1). Only 5 (5.5\%) of GGNs increased in size (Figure 2). There was no significant difference between the average diameter, attenuation value, volume, and mass of the GGNs between CT1 and CT2 (Table 3).

\section{Discussion}

Many GGNs are detected during follow-up CT after resection of the primary lung cancer. GGNs encompass a diverse spectrum of benign and malignant disease, and existing management recommendations for GGNs are based on nodule size, features, and growth rate. There is currently no consensus for the optimal management of concurrent GGNs identified during preoperative or postoperative follow-up CT after the resection of primary non-small cell lung cancer. A history of primary lung cancer is a well-established predictor of GGN growth $(23,24)$. Barsky et al. $(25)$ demonstrated the increasing incidence of lepidic-type tumors, with $25 \%$ showing multifocality; most of these tumors have shown independent clonality upon genetic analyses. Most patients with lung cancer who present with multiple nodules, particularly multiple GGNs or part-solid lesions, actually have synchronous primary carcinomas. One study suggests 

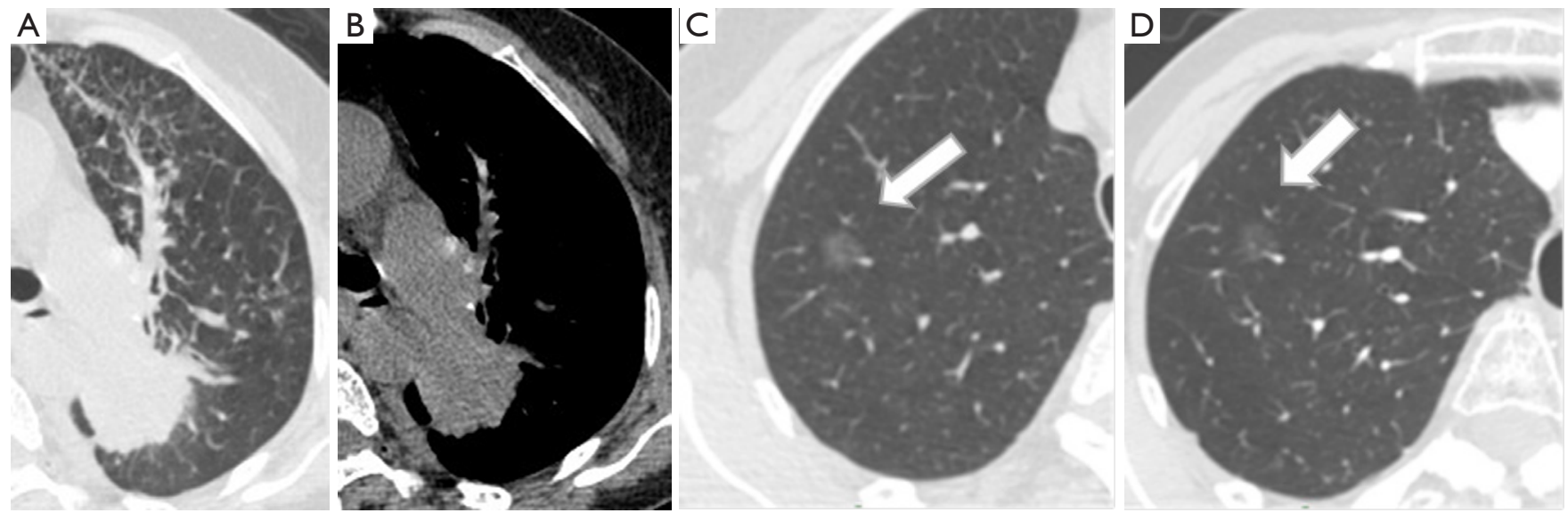

Figure 1 A 74-year-old woman with adenocarcinoma. (A, B) Pre-operation CT scans show a mass and multiple variable-sized nodule in left upper lobe; (C) baseline thin-section CT scan shows a 11-mm well defined pure ground glass nodule (arrow) in right upper lobe; (D) followup thin-section CT scan after 5 cycles cisplatin-based chemotherapy obtains 1 year shows unchangeableness of this pure ground glass nodule seen in (C). CT, computed tomography.
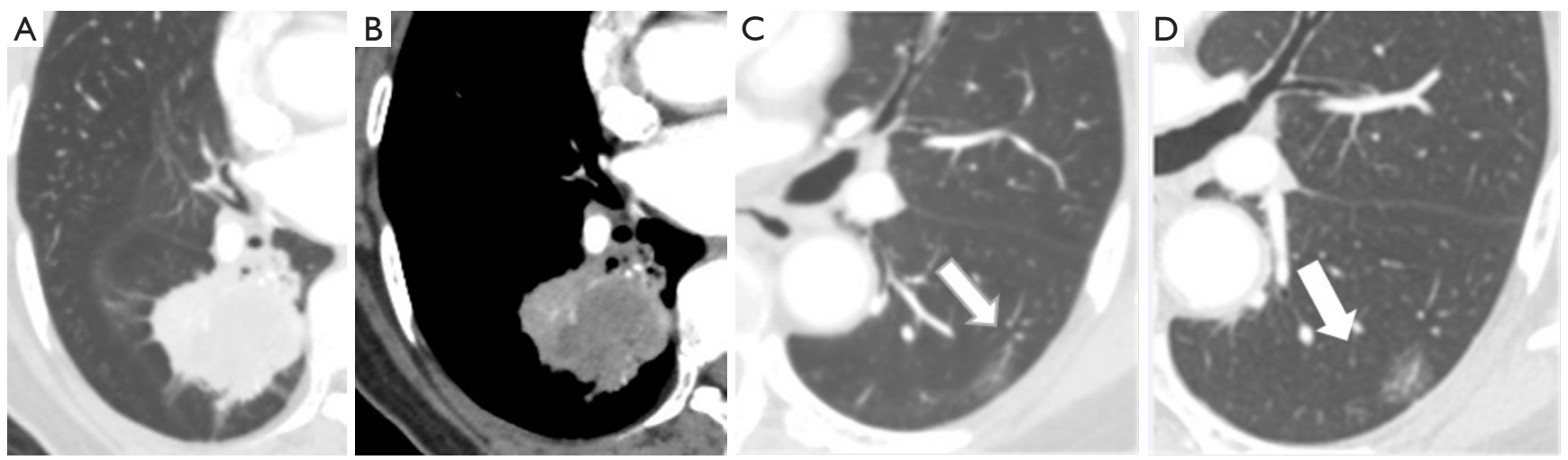

Figure 2 A 47-year-old woman with adenocarcinoma. (A,B) Pre-operation CT scans show a mass in right lower lobe; (C) baseline thinsection CT scan shows a 7-mm well defined pure ground glass nodule (arrow) in left lower lobe; (D) follow-up thin-section CT scan after 7 cycles carboplatin-based chemotherapy obtains 1.5 years shows increasing in size of this pure ground glass nodule seen in (C). CT, computed tomography.

Table 3 Effect of treatment of GGN size, CT attenuation value, volume, and mass

\begin{tabular}{lccc}
\hline Group & Pre-treatment $(\mathrm{CT} 1)$ & Post-treatment $(\mathrm{CT} 2)$ & $\mathrm{P}$ \\
\hline Average size $(\mathrm{mm})$ & $7.61 \pm 3.53$ & $7.86 \pm 3.83$ & 0.055 \\
CT attenuation value $(\mathrm{HU})$ & $-650 \pm 114$ & $-651 \pm 112$ & 0.980 \\
Volume $\left(\mathrm{mm}^{3}\right)$ & $418 \pm 591$ & $467 \pm 678$ & 0.099 \\
Mass $(\mathrm{g})$ & $0.17 \pm 0.28$ & $0.18 \pm 0.28$ & 0.202 \\
\hline
\end{tabular}

GGN, ground glass nodule; CT, computed tomography; HU, Hounsfield unit. 
that patients with at least one GGN tumor on thinsection CT, may have more than three lung cancers at the second surgery and a higher likelihood of new lesions emerging in the future $(26,27)$. The management of synchronous multiple primary lung cancer has been a particular concern for clinicians because the detection of multiple GGNs has been steadily increasing in frequency $(26,27)$.

For patients with multiple GGNs who undergo resection of a dominant GGN, any of the residual GGNs may continue to grow and even evolve into an invasive adenocarcinoma. The frequency of pure GGN growth in the literature is in the range of $0-46 \%(23,27-30)$. Several authors have reported that a history of lung cancer, attenuation value, initial nodule size, and a newly developed solid portion can be helpful in predicting future GGN growth $(23,28,29)$. Chang et al. $(28)$ demonstrated that $91.7 \%$ of growing GGNs were confirmed as lung cancer upon surgical pathology. Cho et al. (30) showed that a persistent, stable, and pure GGNs have a 59\% chance of being malignant. These residual or new GGNs may require additional surgery in the future. In principle, especially for GGN dominant tumors, limited resection should be performed during the second surgery to preserve lung function, given the high likelihood of a third lung cancer developing (26). Traditionally, the decision to resect a lung nodule is determined by CT findings, nodule location, estimated postoperative respiratory function, preoperative comorbidities, and the surgeon's judgment. One study reported the possibility of late recurrence after limited resection, even for small GGNs (13). Surgical resection may impact postoperative respiratory function, especially for older patients who have already undergone a previous lung resection surgery and are unable to tolerate further reduction in lung function. No standard criteria have been established for the selection of the lesions to be treated, nor the method of management of the residual nodules in cases of synchronous multifocal GGNs. The residual or new multiple lung nodules pose a dilemma in clinical management. It is important further to evaluate the clinicopathological features and long-term outcomes of patients with multifocal GGNs, and the optimal management for the residual and new lesions. We evaluated whether medical treatment such as chemotherapy would have any effect on persistent GGNs. In our study, we found that cisplatin or carboplatin based chemotherapy may have no effect on the growth of GGNs. Most GGNs remain stable $(94.5 \%)$ or even increased in size $(5.5 \%)$ after multiple cycles of chemotherapy. This observation is concordant with the mode of action of platinum based chemotherapy agents. Cisplatin and Carboplatin both bind to DNA and inhibit DNA replication, causing cell cycle arrest and programmed cell death. For slow growing tumors that do not replicate rapidly such as adenocarcinoma in situ, these platinum based agents would have a diminished effect. Only four mGGNs were observed in our study, there were no changes either in volume or in density before and after chemotherapy.

Our study had several limitations. First, the small number of patients analyzed and the retrospective nature of the analysis may have affected our results. The small sample size may not have enough statistical power to detect a small improvement in treatment response. Second, we didn't correlate CT findings with pathology. It is possible that some GGN were postinflammatory rather than neoplastic in etiology, although the documented persistence for greater than 3 months makes a postinflammatory process less likely. Furthermore, it would not be feasible in a research study to resect every GGN for histologic analysis as it would adversely affect patient care in most cases. A third limitation of our study was that the mean CT attenuation value of GGNs was evaluated only in the slice containing the part of the lesion with its average diameter. This selected value could potentially be quite different from that of the entire tumor. A fourth limitation is that our 6-month follow-up period and minimum of two chemotherapy cycles may be inadequate to reveal any effect of chemotherapy given the indolent course of the GGNs in this study. Lastly, the image analysis was performed by two radiologists in consensus, and thus, no interobserver agreement on thin-section CT features of GGN could be determined. It is expected that computer-based image post-processing will improve the analysis consistency $(31,32)$.

The proportions of atypical adenomatous hyperplasia (AAH), AIS, minimally invasive adenocarcinoma (MIA), lepidic predominant adenocarcinoma (LPA) and invasive mucinous adenocarcinoma differed for multiple and solitary GGNs. It has been proposed that multiple GGNs have a more indolent nature, because AAH is a preneoplastic lesion and AIS has an excellent prognosis after surgical resection (up to 100\%) (33-35). Kim et al. (36) also report that although the majority of growing GGNs were adenocarcinoma, the clinical course seemed to be indolent. Our study further demonstrates that due to the natural course of the GGNs, chemotherapy may have no effect on GGNs. 


\section{Acknowledgements}

Funding: The project supported by the National Natural Science Foundation of China (Grant No. 81171344).

\section{Footnote}

Conflicts of Interest: The authors have no conflicts of interest to declare.

Ethical Statement: The institutional review boards of our hospitals approved this retrospective study and informed consent was waived.

\section{References}

1. Nakajima R, Yokose T, Kakinuma R, et al. Localized pure ground-glass opacity on high-resolution CT: histologic characteristics. J Comput Assist Tomogr 2002;26:323-9.

2. Austin JH, Müller NL, Friedman PJ, et al. Glossary of terms for CT of the lungs: recommendations of the Nomenclature Committee of the Fleischner Society. Radiology 1996;200:327-31.

3. Naidich DP, Bankier AA, MacMahon H, et al. Recommendations for the management of subsolid pulmonary nodules detected at CT: a statement from the Fleischner Society. Radiology 2013;266:304-17.

4. Raad RA, Suh J, Harari S, et al. Nodule characterization:subsolid nodules. Radiol Clin North Am 2014;52:47-67.

5. Henschke CI, Yankelevitz DF, Mirtcheva R, et al; ELCAP Group. CT screening for lung cancer: frequency and significance of part-solid and nonsolid nodules. AJR Am J Roentgenol 2002;178:1053-7.

6. Hasegawa M, Sone S, Takashima S, et al. Growth rate of small lung cancers detected on mass CT screening. Br J Radiol 2000;73:1252-9.

7. Sone S, Takashima S, Li F, et al. Mass screening for lung cancer with mobile spiral computed tomography scanner. Lancet 1998;351:1242-5.

8. Sobue T, Moriyama N, Kaneko M, et al. Screening for lung cancer with low-dose helical computed tomography: anti-lung cancer association project. J Clin Oncol 2002;20:911-20.

9. Wang YX, Gong JS, Suzuki K, Morcos SK. Evidence based imaging strategies for solitary pulmonary nodule. J Thorac Dis 2014;6:872-87.

10. Lee CT. What do we know about ground-glass opacity nodules in the lung? Transl Lung Cancer Res 2015;4:656-9.

11. Hiramatsu M, Inagaki T, Inagaki T, et al. Pulmonary ground-glass opacity (GGO) lesions-large size and a history of lung cancer are risk factors for growth. J Thorac Oncol 2008;3:1245-50.

12. Takeuchi A, Tsuchida M, Hashimoto T, et al. Segmentectomy for multiple adenocarcinoma presenting as ground-glass opacities after lung cancer surgery. Gen Thorac Cardiovasc Surg 2008;56:410-2.

13. Yoshida J, Ishii G, Yokose T, et al. Possible delayed cut-end recurrence after limited resection for ground-glass opacity adenocarcinoma, intraoperatively diagnosed as Noguchi type B, in three patients. J Thorac Oncol 2010;5:546-50.

14. Aoki T, Nakata H, Watanabe H, et al. Evolution of peripheral lung adenocarcinomas: CT findings correlated with histology and tumor doubling time. AJR Am J Roentgenol 2000;174:763-8.

15. Veronesi G, Bellomi M, Veronesi U, et al.Role of positron emission tomography scanning in the management of lung nodules detected at baseline computed tomography screening. Ann Thorac Surg 2007;84:959-65; discussion 965-6.

16. Travis WD, Brambilla E, Noguchi M, et al. International Association for the Study of Lung Cancer/American Thoracic Society/European Respiratory Society: international multidisciplinary classification of lung adenocarcinoma: executive summary. Proc Am Thorac Soc 2011;8:381-5.

17. Noguchi M, Morikawa A, Kawasaki M, et al. Small adenocarcinoma of the lung. Histologic characteristics and prognosis. Cancer 1995;75:2844-52.

18. Ohtaka K, Hida Y, Kaga K, et al. Limited resection and two-staged lobectomy for non-small cell lung cancer with ground-glass opacity. J Cardiothorac Surg 2013;8:111.

19. Kitahara A, Aoki T, Yazawa M. Surgical treatment for 2 nd primary lung cancer originated close to the initial surgical margin for bronchioloalveolar carcinoma (BAC); report of a case. Kyobu Geka 2013;66:165-8.

20. Nakao M, Yoshida J, Goto K, et al. Long-term outcomes of 50 cases of limited-resection trial for pulmonary groundglass opacity nodules. J Thorac Oncol 2012;7:1563-6.

21. de Hoop B, Gietema H, van de Vorst S, et al. Pulmonary ground-glass nodules: increase in mass as an early indicator of growth. Radiology 2010;255:199-206.

22. Kakinuma R, Ashizawa K, Kuriyama K, et al. Measurement of focal ground-glass opacity diameters on CT images: interobserver agreement in regard to identifying increases in the size of ground-glass opacities. Acad Radiol 
2012;19:389-94.

23. Matsuguma H, Mori K, Nakahara R, et al. Characteristics of subsolid pulmonary nodules showing growth during follow-up with CT scanning. Chest 2013;143:436-43.

24. Kodama K, Higashiyama M, Yokouchi H, et al. Natural history of pure ground-glass opacity after long-term follow-up of more than 2 years. Ann Thorac Surg 2002;73:386-92; discussion 392-3.

25. Barsky SH, Grossman DA, Ho J, et al. The multifocality of bronchioloalveolar lung carcinoma: evidence and implications of a multiclonal origin. Mod Pathol 1994;7:633-40.

26. Hattori A, Suzuki K, Takamochi K, et al. Clinical features of multiple lung cancers based on thin-section computed tomography: what are the appropriate surgical strategies for second lung cancers? Surg Today 2015;45:189-96.

27. Kim HK, Choi YS, Kim J, et al. Management of multiple pure ground-glass opacity lesions in patients with bronchioloalveolar carcinoma. J Thorac Oncol 2010;5:206-10.

28. Chang B, Hwang JH, Choi YH, et al. Natural history of pure ground-glass opacity lung nodules detected by lowdose CT scan. Chest 2013;143:172-8.

29. Tamura M, Shimizu Y, Yamamoto T, et al. Predictive value of one-dimensional mean computed tomography value of ground-glass opacity on high-resolution images for the possibility of future change. J Thorac Oncol 2014;9:469-72.

Cite this article as: $\mathrm{Lu} \mathrm{W}$, Cham MD, Qi L, Wang J, Tang W, Li X, Zhang J. The impact of chemotherapy on persistent ground-glass nodules in patients with lung adenocarcinoma. J Thorac Dis 2017;9(11):4743-4749. doi: 10.21037/ jtd.2017.10.50
30. Cho S, Yang H, Kim K, et al. Pathology and prognosis of persistent stable pure ground-glass opacity nodules after surgical resection. Ann Thorac Surg 2013;96:1190-5.

31. Cavalcanti PG, Shirani S, Scharcanski J, et al. Lung nodule segmentation in chest computed tomography using a novel background estimation method. Quant Imaging Med Surg 2016;6:16-24.

32. Çetinçakmak MG, Göya C, Hamidi C, et al. Quantitative volumetric assessment of pulmonary involvement in patients with systemic sclerosis. Quant Imaging Med Surg 2016;6:50-6.

33. Watanabe S, Watanabe T, Arai K, Kasai T, et al. Results of wedge resection for focal bronchioloalveolar carcinoma showing pure ground-glass attenuation on computed tomography. Ann Thorac Surg 2002;73:1071-5.

34. Kodama K, Higashiyama M, Yokouchi H, et al. Prognostic value of ground-glass opacity found in small lung adenocarcinoma on high-resolution CT scanning. Lung Cancer 2001;33:17-25.

35. Takashima S, Maruyama Y, Hasegawa M, et al. Prognostic significance of high-resolution CT findings in small peripheral adenocarcinoma of the lung: a retrospective study on 64 patients. Lung Cancer 2002;36:289-95.

36. Kim HS, Lee HJ, Jeon JH, et al. Natural history of ground-glass nodules detected on the chest computed tomography scan after major lung resection. Ann Thorac Surg 2013;96:1952-7. 